\title{
Intra-Axial Primary Brain Tumors
}

MRI provides the greatest sensitivity for the detection of brain tumors and information regarding detailed anatomical location and extent, and often elucidates important underlying histopathologic features (Atlas and Lavi, 1996). The majority of primary brain tumors in adults are found in the supratentorial compartment, while tumors in pediatric patients are infratentorial in location. This unit presents the set of sequences used for imaging all types of primary intra-axial brain tumors, whether infiltrative (i.e., astrocytoma, oligodendroglioma, lymphoma) or circumscribed (i.e., ganglioglioma, cystic astrocytoma), and specific modifications will be discussed where necessary. The sequences described in this unit are based on the authors' experience with a 1.5 T scanner (Echospeed GE Medical Systems), but can be expected to be equally applicable to other field strengths and scanners from other manufacturers.

\section{RULE OUT (R/O) PRIMARY CEREBRAL TUMOR}

Spin-echo and fast spin-echo imaging essentially provide all of the information needed to make an accurate diagnosis when evaluating for primary brain tumors. Contrast-enhanced imaging is a critical part of the MR evaluation of brain tumors. The non-contrast spin-echo and fast-spin echo images (sequences 2 and 3) provide information regarding tissue characteristics and extent of tumor. Fluid attenuated inversion recovery (FLAIR) images (sequence 4 ) and the post-contrast $T_{1}$-weighted images (sequence 5) are most sensitive for evaluating spread of tumor to the adjacent meninges (Singer, 1998). Enhancement of a brain lesion indicates breakdown of the blood-brain barrier and non-enhancing portions of a lesion may indicate intra-tumoral cysts or necrosis. Sequences 1 to 5 comprise the preferred protocol.

Gradient echo images depict regions of hypointensity within a tumor that may represent either calcification, blood products or intra-tumoral vessels (Atlas et al., 1988). Gradient echo images (sequence 6) are added on to the Basic Protocol described in this unit when tumors that calcify (i.e., oligodendroglioma, ganglioglioma) or hemorrhage (high grade gliomas, ependymomas) are suspected.

Table A3.3.1 lists the hardware necessary to perform the procedure, along with appropriate parameters.

NOTE: Be sure that technologists and nurses have immediate access to any equipment such as crash carts or oxygen that may be necessary in the event of an emergency. Contrast reactions are rare, but the resources are necessary.

\section{Materials}

Intravenous contrast agent (e.g., Magnevist, Omniscan, or Prohance)

Normal saline $(0.9 \% \mathrm{NaCl})$, sterile

Table A3.3.1 Equipment Parameters for Imaging Brain Tumors

\begin{tabular}{ll}
\hline Coil Type & Quadrature head coil \\
Gradient coil strength & $25 \mathrm{mT} / \mathrm{m}$ (or whatever the system permits) \\
Cardiac gating & No \\
Peripheral gating & For safety only \\
Respiratory gating & No \\
Respirator & If required by patient \\
Oxygen & If required by patient \\
Motion cushions & No
\end{tabular}

Contributed by Annette O. Nusbaum and Scott W. Atlas

Current Protocols in Magnetic Resonance Imaging (2001) A3.3.1-A3.3.10

Copyright $\odot 2001$ by John Wiley \& Sons, Inc.
BASIC

PROTOCOL 1

Cerebral Neoplastic Disease

A3.3.1 


\section{Set up patient and equipment}

1. Interview (screen) the patient to ensure that he or she has no contraindications to the MRI examination, such as a cardiac pacemaker or other implants containing ferromagnetic materials. Question the patient regarding any health conditions that may require the presence of specific emergency equipment during the scanning procedure, or necessitate any other precautions.

Generally standard screening forms are used for all patients scanned in a magnetic resonance system.

The presence of any ferromagnetic metals may be a health hazard to the patient when he or she is inside the magnet, and will also affect the imaging. If in doubt as to the exact composition of the items, it is best to exclude patients with any metal implants; see Shellock (1996) for discussion of what implants may be safely scanned using magnetic resonance.

Patients may be accompanied into the magnet room by a friend or family member, who can sit in the room during the scan and comfort the patient as needed. This companion must be screened as well to ensure the absence of loose metal objects on the body or clothing.

2. If the procedure is a research protocol, have the patient sign any necessary consent form.

3. Have the patient remove all jewelry and change into a gown to eliminate any metal that might be found in clothing.

4. Have the patient wash off any mascara and other makeup to avoid local tissue heating and image artifacts.

5. Inform the patient about what will occur during the procedure, what he or she will experience in the magnet, and how to behave, including the following:

a. If earphones or headphones are used to protect the ears from the loud sounds produced by the gradients, the patient will be asked to wear these, but will be able to communicate with you at any time during the imaging.

b. The patient will be given a safety squeeze-bulb or similar equipment to request assistance at any time (demonstrate how this works).

c. For good results the patient should not talk, and should avoid or minimize swallowing or other movement, during each scan-i.e., as long as the banging sounds continue. Between scans, talking and swallowing are allowed in most cases, but should be avoided when comparative positional studies are being performed; the patient will be informed when this is the case.

d. Nevertheless, the patient may call out at any time if he or she feels it necessary.

6. Have the patient lie in the supine position on the table. Set up all monitoring equipment before or right after the patient lies down.

7. Center the patient in the head coil at the region where the key information is desired. Make sure the head and neck are constrained to prevent motion.

Generally the patient's head is fixed so that the head is horizontal (not tilted) and the neck and head lie along the axis of the patient table.

8. If needed, place a pillow or other support under the knees to make the patient more comfortable.

9. Use the centering light to position the patient (centered on the nasion) and put him or her into the center of the magnet. 
Once this step has been performed, so long as the patient does not move on the table, the table itself can be moved and then replaced in the same position as before without jeopardizing the positioning of one scan relative to another.

10. If the patient is unable to hold still, provide an appropriate sedative.

\section{Sequence 1: Localizer}

11. Run sequence 1 according to Table A3.3.2. The sagittal scout view is used to prescribe the transverse and coronal planes.

\section{Sequence 2: $T_{1}$-weighted scan}

12. Run sequence 2 according to Table A3.3.3.

\section{Sequence 3: $T_{2}$-weighted scan}

13. Run sequence 3 according to Table A3.3.4.

\section{Sequence 4: Fast fluid attenuated inversion recovery (FLAIR)}

14. Run sequence 4 according to Table A3.3.5.

\section{Sequence 5: Post-contrast imaging}

15. Remove the patient from the scanner. The patient should not move on the table. Establish an intravenous line from which the contrast agent can be injected, and attach this line securely to the patient so that movement into or out of the magnet will not pull at the patient's arm. Move the patient back into the scanner.

It is preferable to insert the line prior to imaging and to leave the patient in the magnet, with no intervening motion, between the scans run before contrast agent injection and those run after injection.

16. Leaving the patient in the magnet, inject the contrast agent, flush the line with $10 \mathrm{ml}$ saline.

A dose of $0.1 \mathrm{mmol} / \mathrm{kg}$ of contrast agent is usually given.

A delay in scanning may actually be beneficial when evaluating for metastases, which is one of the reasons we scan in multiple planes after intravenous contrast administration.

Table A3.3.2 Primary Clinical Imaging Parameters for Sequence 1: Localizer ( $T_{1}$-Weighted)

\begin{tabular}{ll}
\hline Patient position & Supine \\
Scan type & Spin echo \\
Imaging plane (orientation) & Sagittal \\
Central slice or volume center & Laser light centered on nasion \\
Echo time $\left(T_{\mathrm{E}}\right)$ & $11 \mathrm{msec}$ (or select "minimum \\
& full" echo time) \\
Receiver bandwidth $(\mathrm{RBW})$ & $10 \mathrm{kHz}$ \\
Repeat time $\left(T_{\mathrm{R}}\right)$ & $500 \mathrm{msec}$ \\
Flip angle $(\mathrm{FA})$ & $90^{\circ}$ \\
Fields of view $(\mathrm{FOV}, \mathrm{xOV}$ & $240 \mathrm{~mm}, 240 \mathrm{~mm}$ \\
Resolution $(\Delta x, \Delta y)$ & $0.94 \mathrm{~mm}, 1.25 \mathrm{~mm}$ \\
Number of data points collected, $\left(N_{\mathrm{x}}, N_{\mathrm{y}}\right)$ & 256,192 \\
Slice thickness $(\Delta z)$ & $5 \mathrm{~mm}$ \\
Number of slices & $20 \mathrm{or}$ as many as needed to cover \\
Slice gap & the region of interest \\
Number of acquisitions $\left(N_{\text {acq }}\right)$ & $2 \mathrm{~mm}$ \\
Swap read and phase encoding & 1 \\
Saturation pulses & No \\
Scan time & Not applicable \\
\hline
\end{tabular}

Cerebral Neoplastic Disease

A3.3.3 
Table A3.3.3 Primary Clinical Imaging Parameters for Sequence 2 ( $T_{1}$-Weighted)

$\begin{array}{ll}\text { Patient position } & \text { Supine } \\ \text { Scan type } & \text { Spin echo } \\ \text { Imaging plane (orientation) } & \text { Transverse } \\ \text { Central slice or volume center } & \text { Laser light centered on nasion } \\ \text { Echo time }\left(T_{\mathrm{E}}\right) & \begin{array}{l}11 \mathrm{msec}(\text { or select "minimum } \\ \text { full" echo time) }\end{array} \\ & 10 \mathrm{kHz} \\ \text { Receiver bandwidth }(\mathrm{RBW}) & 500 \mathrm{msec} \\ \text { Repeat time }\left(T_{\mathrm{R}}\right) & 90^{\circ} \\ \text { Flip angle }(\mathrm{FA}) & 240 \mathrm{~mm}, 240 \mathrm{~mm} \\ \text { Fields of view }\left(\mathrm{FOV}_{\mathrm{x}}, \mathrm{FOV}_{\mathrm{y}}\right) & 0.94 \mathrm{~mm}, 1.25 \mathrm{~mm} \\ \text { Resolution }(\Delta x, \Delta y) & 256,192 \\ \text { Number of data points collected, }\left(N_{\mathrm{x}}, N_{\mathrm{y}}\right) & 5 \mathrm{~mm} \\ \text { Slice thickness }(\Delta z) & 20 \text { or as many as needed to cover } \\ \text { Number of slices } & \text { the region of interest } \\ & 2.5 \mathrm{~mm} \\ \text { Slice gap } & 1 \\ \text { Number of acquisitions }\left(N_{\mathrm{acq}}\right) & \text { Yes } \\ \text { Swap read and phase encoding } & \text { Not applicable } \\ \text { Saturation pulses } & 1 \mathrm{~min}, 36 \text { sec } \\ \text { Scan time } & \end{array}$

Table A3.3.4 Primary Clinical Imaging Parameters for Sequence 3 ( $T_{2}$-Weighted)

Patient position

Scan type

Imaging plane (orientation)

Central slice or volume center

Echo time $\left(T_{\mathrm{E}}\right)$

Receiver bandwidth (RBW)

Echo train length (ETL)

Repeat time $\left(T_{\mathrm{R}}\right)$

Flip angle (FA)

Fields of view $\left(\mathrm{FOV}_{\mathrm{x}}, \mathrm{FOV}_{\mathrm{y}}\right)$

Resolution $(\Delta x, \Delta y)$

Number of data points collected, $\left(N_{\mathrm{x}}, N_{\mathrm{y}}\right)$

Slice thickness $(\Delta z)$

Number of slices

Slice gap

Number of acquisitions ( $N_{\text {acq }}$ )

Swap read and phase encoding

Saturation pulses

Scan time
Supine

Fast spin echo

Transverse

Laser light centered on nasion

$102 \mathrm{msec}$ (effective)

$16 \mathrm{kHz}$

8

$3600 \mathrm{msec}$

$90^{\circ}$

$240 \mathrm{~mm}, 240 \mathrm{~mm}$

$0.94 \mathrm{~mm}, 1.25 \mathrm{~mm}$

256, 192

$5 \mathrm{~mm}$

20 or as many as needed to cover the region of interest

$2.5 \mathrm{~mm}$

1

Yes

Not applicable

$\sim 1 \mathrm{~min}, 30 \mathrm{sec}$

Intra-Axia 
17. Acquire the post-contrast images using the same parameters as in sequence 2 ( $T_{1}$-weighted).

In addition to the transverse plane, the coronal plane is routinely obtained, with the following changes to the parameters in sequence 2: (a) flow compensation is on; (b) $T_{E}$ is 15 msec (prolonged due to flow compensation gradients); and (c) it is not necessary to

Table A3.3.5 Primary Clinical Imaging Parameters for Sequence 4 (Fast FLAIR)

\begin{tabular}{ll}
\hline Patient position & Supine \\
Scan type & Inversion recovery fast spin echo \\
Imaging plane (orientation) & Transverse \\
Central slice or volume center & Laser light centered on nasion \\
Echo time $\left(T_{\mathrm{E}}\right)$ & $120 \mathrm{msec}$ (effective) \\
Receiver bandwidth (RBW) & $16 \mathrm{kHz}$ \\
Echo train length (ETL) & 8 \\
Repeat time $\left(T_{\mathrm{R}}\right)$ & $10,000 \mathrm{msec}$ \\
Inversion time $\left(T_{\mathrm{I}}\right)$ & $2200 \mathrm{msec}$ \\
Flip angle $(\mathrm{FA})$ & $180^{\circ}$ \\
Fields of view $(\mathrm{FOV}, \mathrm{x}$, FOV & $\mathrm{y})$ \\
Resolution $(\Delta x, \Delta y)$ & $240 \mathrm{~mm}, 240 \mathrm{~mm}$ \\
Number of data points collected, $\left(N_{\mathrm{x}}, N_{\mathrm{y}}\right)$ & $0.94 \mathrm{~mm}, 1.25 \mathrm{~mm}$ \\
Slice thickness $(\Delta z)$ & 256,192 \\
Number of slices & $5 \mathrm{~mm}$ \\
& 20 or as many as needed to cover \\
Slice gap & the region of interest \\
Number of acquisitions $\left(N_{\text {acq }}\right)$ & $2.5 \mathrm{~mm}$ \\
Swap read and phase encoding & 1 \\
Saturation pulses & Yes \\
Scan time & Not applicable \\
\hline
\end{tabular}

Table A3.3.6 Primary Clinical Imaging Parameters for Sequence 6

\begin{tabular}{ll}
\hline Patient position & Supine \\
Scan type & 2-D gradient recalled echo \\
Imaging plane (orientation) & Transverse \\
Central slice or volume center & Laser light centered on nasion \\
Echo time $\left(T_{\mathrm{E}}\right)$ & $30 \mathrm{msec}$ \\
Receiver bandwidth $(\mathrm{RBW})$ & $4 \mathrm{kHz}$ \\
Repeat time $\left(T_{\mathrm{R}}\right)$ & $500 \mathrm{msec}$ \\
Flip angle $(\mathrm{FA})$ & $15^{\circ}$ \\
Fields of view $\left(\mathrm{FOV}_{\mathrm{x}}, \mathrm{FOV}_{\mathrm{y}}\right)$ & $240 \mathrm{~mm}, 240 \mathrm{~mm}$ \\
Resolution $(\Delta x, \Delta y)$ & $0.94 \mathrm{~mm}, 1.25 \mathrm{~mm}$ \\
Number of data points collected, $\left(N_{\mathrm{x}}, N_{\mathrm{y}}\right)$ & 256,192 \\
Slice thickness $(\Delta z)$ & $5 \mathrm{~mm}$ \\
Number of slices & 20 or as many as needed to cover \\
& the region of interest \\
Slice gap & $2.5 \mathrm{~mm}$ \\
Number of acquisitions $\left(N_{\mathrm{acq}}\right)$ & 1 \\
Swap read and phase encoding & Yes \\
Saturation pulses & Not applicable \\
Scan time & $\sim 2 \mathrm{~min}$ \\
\hline
\end{tabular}

Cerebral Neoplastic Disease

A3.3.5 
ALTERNATE PROTOCOL

Intra-Axial Primary Brain Tumors

A3.3.6 swap read and phase encoding directions. In patients requiring sedation and in all pediatric patients, we also routinely obtain a third post-contrast plane of imaging (sagittal).

\section{Sequence 6: Gradient echo imaging to examine calcified or hemorrhagic tumor}

18. Run sequence 6 according to Table A3.3.6.

Gradient echo images depict regions of hypointensity within tumors that may represent either calcification, blood products, or flow voids from intra-tumoral vessels. This sequence is useful to obtain in lesions that frequently calcify (i.e., oligodendroglioma, ganglioglioma) or hemorrhage (i.e., high grade gliomas and ependymomas).

\section{OPTIONAL SEQUENCES}

The set of imaging sequences described in Basic Protocol 1 often provide virtually all the information necessary for the diagnosis of primary intra-axial brain tumors. Studies are being conducted to investigate the potential value of diffusion-weighted imaging (DWI; Stejskal and Tanner, 1965; Tien et al., 1994) and perfusion-weighted imaging (Alsop and Detre, 1998; using either contrast bolus techniques or continuous arterial spin labeling) in routine tumor imaging. Preliminary studies suggest that DWI may have potential for detecting intra-tumoral necrosis.

The main indication for obtaining proton MR spectroscopy (MRS) data is to help distinguish a neoplastic from a non-neoplastic lesion (see Figure A3.3.1); however, proton MR spectroscopy (sequence 7), does provide biochemical information regarding brain tumors that may be useful to further categorize a specific lesion (i.e., high or low grade tumor) (Meyer et al., 1999). Whole brain chemical-shift imaging (CSI) maps may also be potentially useful in the investigation of brain tumors by providing visual metabolite maps, rather than spectral data.

\section{Set up patient and equipment}

1. Follow the setup for Basic Protocol 1 and obtain the necessary pre- and post-contrast images (steps 1 to 17). The lesion is localized on a transverse image (typically the post-contrast transverse $T_{1}$-weighted image).

Voxel placement requires physician input to ensure the correct region of interest is being studied. Two separate scans are performed: one with the voxel placed over the area in question (typically the enhancing portion of the lesion), the other scan is performed with the voxel placed in the contralateral hemisphere (in a "normal appearing" analogous area of the brain). The screen should always be saved to document placement of the voxel.

\section{Sequence 7: Proton $\left({ }^{1} \mathrm{H}\right)$ MR spectroscopy}

The Proton Brain Exam-Single Voxel (PROBE-SV) on GE systems is a fully automated software package that is widely available and is performed using the single voxel technique $\left(8 \mathrm{~cm}^{3}\right)$. A high field system is required to obtain good spectra. There are 2 possible localization methods: STEAM (stimulated echo acquisition mode) or PRESS (point-resolved spectroscopy). The sequence parameters can be set by the user $\left(T_{\mathrm{R}}, T_{\mathrm{E}}\right.$, voxel size). Short $T_{\mathrm{E}}$ spectroscopy has the advantage of providing more information, since more peaks are visible for analysis while maximizing the detection of lipid-breakdown products. Long- $T_{\mathrm{E}}$ spectra have less baseline distortion and are easier to quantify. Since there is a $\sqrt{2}$ decrease in the signal-to-noise ratio with STEAM sequences, the PRESS sequence is usually preferred. Spectra obtained following the administration of intravenous contrast may be altered (Sijens et al., 1997). In particular, studies have shown a mean loss of $15 \%$ of the peak area of choline-containing compounds without significant changes in creatine (Cre) or N-acetyl aspartate (NAA) (Sijens et al., 1997). In routine clinical practice, the spectra are obtained following the administration of intravenous contrast and 
the voxel is positioned over the solid (enhancing) portion of the lesion so that theoretically signal is acquired from viable tumor tissue.

2. Perform automated shimming and water suppression, center the voxel over the enhancing portion of the lesion, and run sequence 7 according to Table A3.3.7.

3. After completion of the scan, a spectrum will appear on the screen and the values for the spectrum should be saved.

4. Place the voxel in the contralateral hemisphere and repeat the scan.

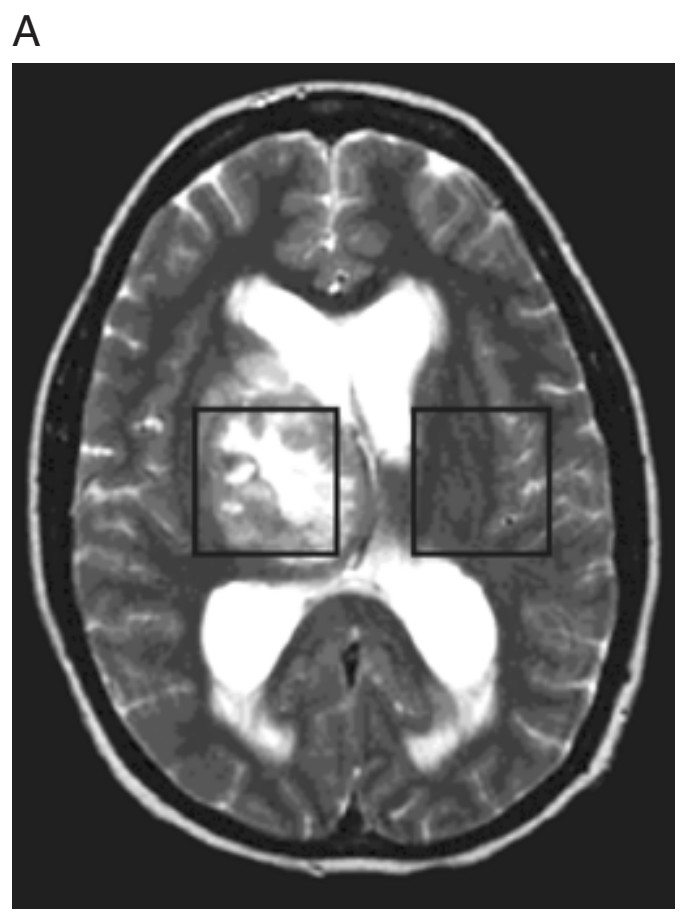

B

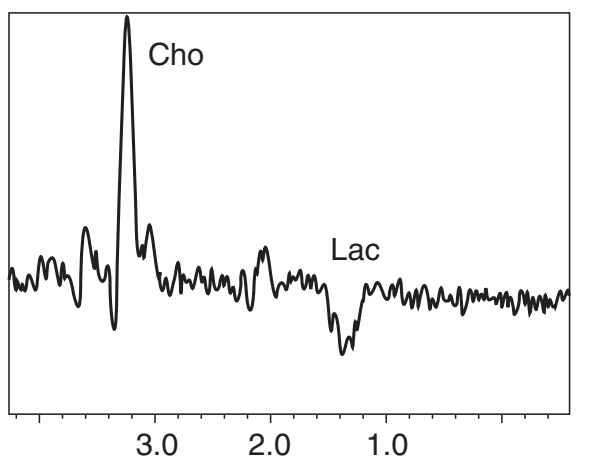

C

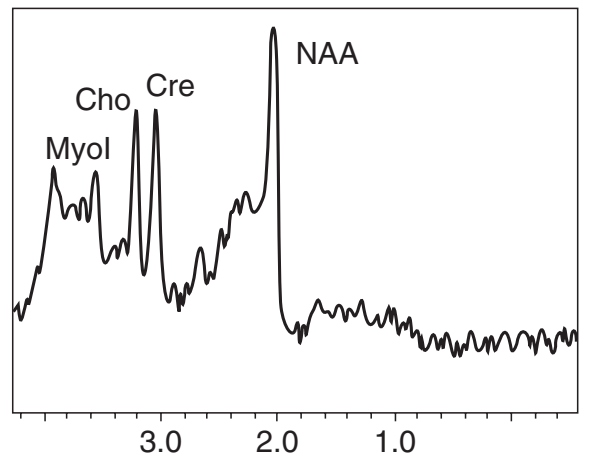

Figure A3.3.1 (A) $T_{2}$-weighted fast spin echo image $\left(T_{\mathrm{R}}=2000 \mathrm{msec}, T_{\mathrm{E}}=105 \mathrm{msec}, N_{\text {acq }}=2\right)$ through a large mass in the right thalamus and basal ganglia of a 35 year old female with headaches. Water suppressed protocon spectroscopy was performed using PRESS sequence (point resolved spectroscopy) in an 8- $\mathrm{cm}^{3}$ voxel with a $T_{\mathrm{E}}=144 \mathrm{msec}$ positioned (B) over the tumor mass and (C) on the contralateral side at the equivalent location. Resonances are labeled as: Lac = lactate; NAA $=\mathrm{N}$-acetyl aspartate; $\mathrm{Cre}=$ creatine $;$ Cho = choline; $\mathrm{Myol}=$ myoinositol. The horizontal axis is given in parts per million (ppm). Automated software used creatine as reference signal intensity quantified the NAA as significantly decreased in the tumor and NAA/Cre ratio of 1.47 in the normal tissue. The presence of lactate, markedly reduced NAA and elevated choline, is characteristic of neoplasm.

Cerebral Neoplastic Disease 
Table A3.3.7 Primary Clinical Imaging Parameters for Sequence $7\left({ }^{1} \mathrm{H}-\mathrm{MR}\right.$

Spectroscopy)

$\begin{array}{ll}\text { Patient position } & \text { Supine } \\ \text { Scan type } & \text { PROBE-SV } \\ \text { Voxel center } & \text { See text (steps 2 and 4) } \\ \text { Echo time }\left(T_{\mathrm{E}}\right) & 35 \mathrm{msec} \text { and } 144 \mathrm{msec} \\ \text { Repeat time }\left(T_{\mathrm{R}}\right) & 2000 \mathrm{msec} \\ \text { Flip angle }(\mathrm{FA}) & 90^{\circ} \\ \text { Voxel size }(\Delta x, \Delta y, \Delta z) & 2 \mathrm{~cm}, 2 \mathrm{~cm}, 2 \mathrm{~cm} \\ \text { Number of acquisitions }\left(N_{\text {acq }}\right) & 8^{a} \text { and } 128^{b} \\ \text { Saturation pulses } & \text { Water } \\ \text { Scan time } & \sim 5 \mathrm{~min}\end{array}$

${ }^{a}$ Without water suppression.

${ }^{b}$ With water suppression.

Table A3.3.8 Primary Clinical Imaging Parameters for Sequence 8 (3-D Short $T_{\mathrm{R}}$ Gradient Echo)

\begin{tabular}{ll}
\hline Patient position & Supine \\
Scan type & 3-D gradient echo \\
Imaging plane (orientation) & Transverse \\
Central slice or volume center & Laser light centered on nasion \\
Echo time $\left(T_{\mathrm{E}}\right)$ & $5 \mathrm{msec}$ (or select the minimum \\
& value when possible) \\
Repeat time $\left(T_{\mathrm{R}}\right)$ & $35 \mathrm{msec}$ \\
Flip angle $(\mathrm{FA})$ & $45^{\circ}$ \\
Fields of view $\left(\mathrm{FOV}_{\mathrm{x}}, \mathrm{FOV}_{\mathrm{y}}\right)$ & $240 \mathrm{~mm}, 240 \mathrm{~mm}$ \\
Resolution $(\Delta x, \Delta y)$ & $0.94 \mathrm{~mm}, 1.25 \mathrm{~mm}$ \\
Number of data points collected $\left(N_{\mathrm{x}}, N_{\mathrm{y}}\right)$ & 256,192 \\
Slice thickness $(\Delta z)$ & $1.5 \mathrm{~mm}$ \\
Number of slices & $\sim 40$ \\
Slice gap & 0 \\
Number of acquisitions $\left(N_{\mathrm{acq}}\right)$ & 1 \\
Swap read and phase encoding & Yes \\
Saturation pulses & Not applicable \\
Scan time & $\sim 5 \mathrm{~min}$ \\
\hline
\end{tabular}

5. After completion of the scan, another spectrum will appear on the screen and the values for the spectrum should be saved.

\section{Sequence 8: 3-D short $T_{R}$ gradient echo}

6. In cases where it is difficult to differentiate an intra-axial peripheral exophytic tumor from an extra-axial lesion impinging on the brain, obtain a thin slice 3-D gradient echo sequence to better evaluate the brain-tumor interface outlined in Table A3.3.8. 


\section{R/O PRIMARY CEREBELLAR AND BRAINSTEM TUMOR}

In pediatric patients, tumors are most commonly found in the infra-tentorial compartment (posterior fossa). In adults, the majority of lesions found in the posterior fossa are metastases; however, primary tumors do occur and the most common primary cerebellar tumor in adults is hemangioblastoma. The sequences described in Basic Protocol 1 should be obtained to evaluate posterior fossa tumors.

\section{Set up patient and equipment}

1. Follow the setup for Basic Protocol 1 (steps 1 to 10).

2. Run sequences 1 to 5 .

\section{COMMENTARY}

\section{Background Information}

The incidence of brain tumors varies according to age and recent literature suggests incidence trends have stabilized for all age groups except in individuals greater than 85 years where it is increasing (15.7 cases of cancer per 100,000 individuals). Improvements in diagnosis may explain the observed patterns in brain cancer trends (Legler et al., 1991). While computed tomography (CT) is often more readily available, and therefore the first examination performed for suspected intracranial pathology, magnetic resonance imaging (MRI) has proven to be invaluable in refining the diagnosis. MRI provides greater sensitivity for the detection of brain tumors and detailed anatomical information (Atlas and Lavi, 1996). Contrast enhanced imaging in more than one plane is essential to evaluate intra-axial brain tumors.

\section{Critical Parameters and Troubleshooting}

In pediatric patients and other subjects requiring sedation, the authors feel that 3 planes (transverse, coronal, sagittal) of imaging should be obtained in the post-contrast acquisition when evaluating brain tumor patients. Proton MR spectroscopy studies have shown that contrast administration will alter spectra by decreasing the peak area of choline- containing compounds. Since tumors are frequently heterogeneous histologically, placement of the voxel in different regions of the tumor may yield different MR spectroscopy results. The relatively large voxel size also results in volume averaging with the adjacent brain. In routine clinical use, the post-contrast images are used to identify the solid appearing portion of the lesion and the voxel is placed in the solid (enhancing portion) of the lesion.

\section{Anticipated Results}

The goal in evaluating cerebral neoplasms is to image the entire lesion, localize it to the appropriate compartment of the brain, and attempt to predict the underlying histology. Conventional MR sequences offer superb anatomical detail and tissue characterization of brain tumors. Contrast-enhanced imaging is critical to evaluate all brain tumors and helps to assess the integrity of the blood-brain barrier and depict intra-tumoral cystic or necrotic regions. Conventional MR techniques cannot reliably distinguish high-grade from low-grade tumors. Proton MR spectroscopy offers metabolic information and helps predict the relative aggressiveness of a lesion by evaluating metabolite ratios (NAA/Cre) and identifying metabolites such as lactate (indicator of anaerobic glycolysis; Meyer et al., 1999).

\section{Time Considerations}

The Basic Protocol 1 detailed in this unit should take $\sim 30 \mathrm{~min}$ to complete. Proton MR Spectroscopy may add $\sim 10$ to $15 \mathrm{~min}$ to the imaging session.

\section{Literature Cited}

Alsop, D.C. and Detre, J.A. 1998. Multisection cerebral blood flow MR imaging with continuous arterial spin labeling. Radiology 208:410-416.

Atlas, S.W., Mark, A.S., Grossman, R.I., Gomori, J.M. 1988. Intracranial hemorrhage: Gradientecho MR imaging at 1.5 T. Radiology 168:803807.

Atlas, S.W. and Lavi, E. 1996. Intra-axial brain tumors. In Magnetic Resonance Imaging of the Brain and Spine, 2nd Edition. ( S.W. Atlas, ed.) PA.

Legler, J.M., Gloeckler Ries, L.A., Smith, M.A., Warren, M.A., Warren, J.L., Heineman, E.F., Kaplan, R.S., and Linet, M.S. 1991. Brain and other central nervous system cancers: Recent pp. 315-422. Lippincott-Raven, Philadelphia,
Cerebral Neoplastic Disease

A3.3.9 
trends in incidence and mortality. J. Natl. Cancer Inst. 91:1382-1390.

Meyer, M.E., Pipas, J.M., Mamourian, A., Tosteson, T.D., and Dunn, J.F. 1999. Classification of biopsy-confirmed brain tumors using single-voxel MR spectroscopy. Am. J. Neuroradiol. 20:117123.

Shellock, F.G. 1996. Pocket Guide to MR Procedures and Metallic Objects. Lippincott-Raven, Philadelphia.

Sijens, P.E., van den Bent, M.J., Nowak, P.J., van Dijk, P., and Ouderk, M. 1997. ${ }^{1} \mathrm{H}$ chemical shift imaging reveals loss of brain tumor choline signal after administration of Gd-contrast. Magn. Reson. Med. 37:222-225.

Singer, M.B., Atlas, S.W., and Drayer, B.P. 1998. Subarachnoid space disease: Diagnosis with fluid-attenuated inversion-recovery MR imaging and comparison with gadolinium-enhanced spin echo MR imaging-blinded reader study. Radiology 208:417-422.

Stejskal, E.O. and Tanner, J.E. 1965. Spin diffusion measurements: spin echoes in the presence of time-dependent field gradient. J. Chem. Phys. 42:288-292.

Tien, R.D., Flesberg, G.J., Friedman, H., Brown, H., and MacFall, J. 1994. MR imaging of high-grade cerebral gliomas: value of diffusion-weighted echoplanar pulse sequences. Am. J. Roentgenol. 162:671-677.

Contributed by Annette O. Nusbaum

New York Presbyterian Hospital

New York, New York

\section{Scott W. Atlas}

Stanford University Medical Center

Stanford, California
Intra-Axial Primary Brain Tumors

A3.3.10 